\section{Cross-modality matching of muscular tension to loudness*}

\author{
L. J. J. BRUNO $\dagger$, R. F. HEFFERLINE, and P. D. SUSLOWITZ \\ Columbia University, New York, New York 10027
}

In a single session, Ss produced and estimated a series of electromyographically measured tensions, estimated the loudness of pure tones, and then matched muscular tension to tonal loudness. In all cases, power functions adequately represented the estimation and matching data. For two Ss, who scaled tension in the abductor of the little finger, the slopes of the estimation functions successfully predicted the slopes of the matching functions. For the other Ss the prediction from estimation to matching data was fair, but not good; these Ss scaled adductor tension, for which the electromyographic measurements were methodologically questionable. The data suggest that a consistent scale of muscular tension can be constructed, and that the scale can be validated by crossmodal comparison.

Although the reports of Mountcastle (1965) and of Wood (1969) have begun to map out the psychophysics of pressure and movement sensitivity, work on the other "body senses" is notable only for its scarcity (Hefferline, 1962). In particular, little is known of our ability to scale sustained muscular contractions of the sort perceptually interpreted as a manifestation of set (cf. Allport, 1955) and clinically implicated as a factor in functional disorders (cf. Whatmore \& Kohli, 1968).

What is known suggests that perceived tension grows as a power function of the magnitude of muscular contractions. J. C. Stevens and Mack (1959) found that the apparent force exerted in squeezing a hand-held dynamometer was a power function of physical force with an exponent of 1.7. Hefferline (1958) found that Ss trained with feedback to sustain covert contractions (measured electromyographically) later produced without feedback contraction magnitudes that were related to the required magnitudes by a power function with an exponent of about 0.5. These studies, however, require confirmation: J.C. Stevens and Mack did not record the electromyogram; Hefferline's range of tensions was too small.

The present study was undertaken (1) to determine, more precisely, the relationship of perceived tension to the magnitude of sustained muscular contractions, as measured electromyographically, and (2) to validate these findings through cross-modality matching of tension to loudness (cf. S. S. Stevens, 1959). The

* This paper is based on the work described in Electropsychology Report $\mathrm{MH}-13890-07$ and supported by NIMH Grant MH-13890 to R.F. Hefferline, Principal Investigator.

† Address: Electropsychology Laboratory, Columbia University, New York, New York 10027 . study deals with individual tension, loudness, and matching functions, each taken in the same session, and each based on data generated by one determination per point.

\section{EMG TECHNIQUE}

Surface electrodes were used to record the electromyogram (EMG) from the muscles which abduct and adduct the little finger of the right hand. Figure 1 illustrates the electrode placement and depicts the plane and direction for abduction and adduction. The EMG taken from this placement characteristically includes waveforms of long and short durations during virtually all degrees of sustained isometric contraction (Bruno, Davidowitz, \& Hefferline, 1970). Since the long waveforms originate in the abductor and the short waveforms originate in the adductor of the little finger, we were able to validate our recordings by using an oscilloscope to measure waveform duration.

To quantify the EMG, however, another measurement method was needed. We chose to determine EMG magnitude using a rms-reading voltmeter since, by definition, the measurements made in this way are proportional to the area under the curve of the surface EMG, which, under certain limited conditions, is linearly related to the force of voluntary muscular contraction (cf. Lippold, 1952, 1967). Two conditions under which the relationship between the integrated EMG and the force of contraction can be expected to hold are (1) that the contraction is isometric and (2) that the EMG is taken from a muscle which is the only one involved in executing a particular movement. In the present study, both conditions were met during abduction of the little finger, but the second was violated during adduction, since, in addition to the interosseous of the hand, there are forearm muscles which may contribute to adductive movements.

\section{TENSION ESTIMATION}

\section{Subjects}

The Ss were four members of the laboratory staff. All had normal hearing and had no known motor deficits. Two were asked to scale abductive tensions (LJJB, male, 26, right-handed; JACB, female, 23, left-handed) and two to scale adductive tensions (PDS, male, 21, right-handed; JCJB, male, 18, right-handed). Two (LJJB and PDS) were experienced in psychophysical and psychophysiological tasks; the others were not.

\section{Apparatus}

The apparatus used to record and measure the EMG has been described in detail elsewhere (Bruno et al, 1970).

\section{Procedure}

EMG measurements (in decibels relative to the energy produced by a $200-\mathrm{Hz}$ sine wave at 3.5 microV rms amplitude) were made as the $S$ sat in a reclining chair, with his right hand resting palm-down on a padded board which held two small upright bars (see Fig. 1). The tip of his little finger was placed between the bars, which were cushioned with No.9 polyvinylchloride tubing, and the bars were adjusted until they rested lightly against the sides of the finger. Once fixed in position, the bars served both to define the tension required and to limit the $S$ to isometric contractions. ${ }^{1}$

Eight abductive or adductive magnitudes, individually chosen for each $\mathrm{S}$ to preclude extreme values, appeared once each in random order as comparison stimuli. One of these-an intermediate magnitude-was chosen as the modulus and

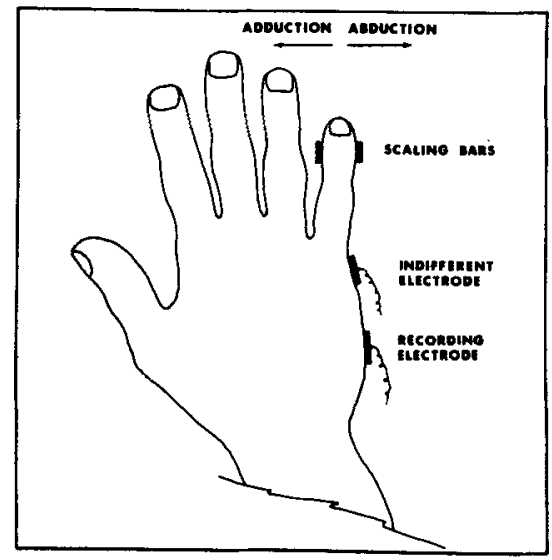

Fig. 1. Electrode placement on the hypothenar eminence, and the position of the scaling bars used to define abductive and adductive tensions. 
Table 1

Power-Function Exponents for Tension Estimation, Loudness Estimation, and Matching of Tension to Loudness

\begin{tabular}{lccccc}
\hline & \multicolumn{2}{c}{ Tension } & & \multicolumn{2}{c}{ Matching } \\
\cline { 2 - 3 } S & Abductive & Adductive & Loudness & Predicted & Obtained \\
\hline LJJB & 1.04 & & 0.58 & 0.56 & 0.53 \\
JACB & 1.09 & & 0.48 & 0.44 & 0.39 \\
PDS & & 1.31 & 0.51 & 0.39 & 0.23 \\
JCJB & 1.33 & 0.62 & 0.47 & 0.30 \\
\hline
\end{tabular}
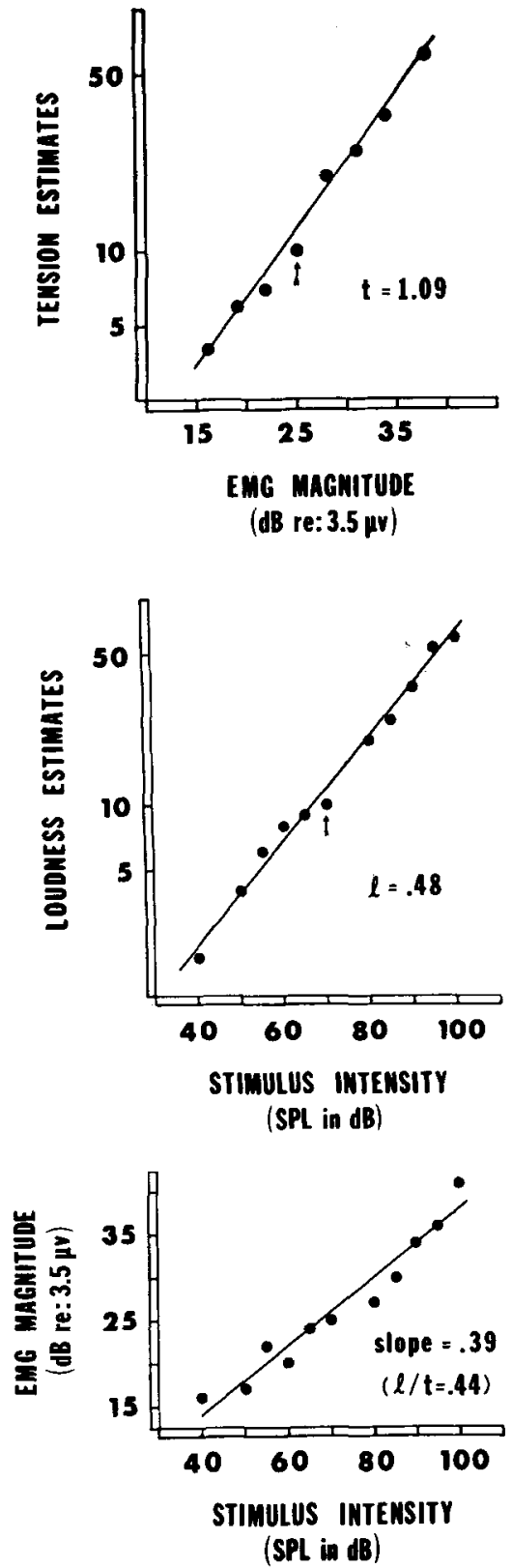

Fig. 2. Top: Magnitude estimation of abductive tension. Middle: Magnitude estimation of the loudness of a $1,000-\mathrm{Hz}$ tone. Bottom: Abductive tension matched to tonal loudness. Arrows mark the moduli; $t, l$, and slope are the slopes of the power functions graphed by the straight lines drawn through the data points. Functions are for an individual $O$ (JACB) giving one determination per point. was first asked to reproduce the modulus, and, if he failed, was corrected through instructions until he succeeded. When the modulus had been sustained for about $0.5 \mathrm{sec}$, the $S$ was asked to relax, and then, after $5 \mathrm{sec}$, he was asked to produce a tension convenient for him. At this point he was instructed to increase or decrease his level of muscular activity until the magnitude achieved through adjustment approximated, as closely as practicable, the magnitude selected as the comparison stimulus for that trial. When the comparison had been held for about $0.5 \mathrm{sec}$, the $\mathrm{S}$ was asked to relax and to report a number which stood in proportion to " 10 " as the comparison tension stood in proportion to the modulus. The next trial began about $30 \mathrm{sec}$ after the S's report.

EMG waveform durations were measured on-line throughout the tension estimation procedure. In the few cases be inappropriate to the required tension, the data of that trial were discarded, and the trial was repeated later in the session.

\section{Results}

Power functions were fitted to the tension estimation data by the method of least squares. The exponents of the functions are given for each $S$ in Table 1, where it can be seen that the exponent for abductive tension-about 1.0-is somewhat smaller than the exponent for adductive tension, which is about 1.3. These findings are in agreement with those previously obtained in our laboratory using the methods of magnitude estimation and magnitude production (Bruno, unpublished data).

The data taken from naive Ss scaling abductive and adductive tensions are presented in the upper panels of Figs. 2 and 3 , respectively. The straight lines drawn through the data points are the graphs of power functions with slopes $(t)$ taken from the least-squares equations, but with intercepts fitted by eye. The data appear to be adequately fitted by the lines, even though each point represents the outcome of but a single trial. The data obtained from experienced Ss differ from those presented here only in that they were somewhat more orderly, and that they spanned a wider range of tensions. assigned the value "10." On each trial the $S$ where waveform durations were found to
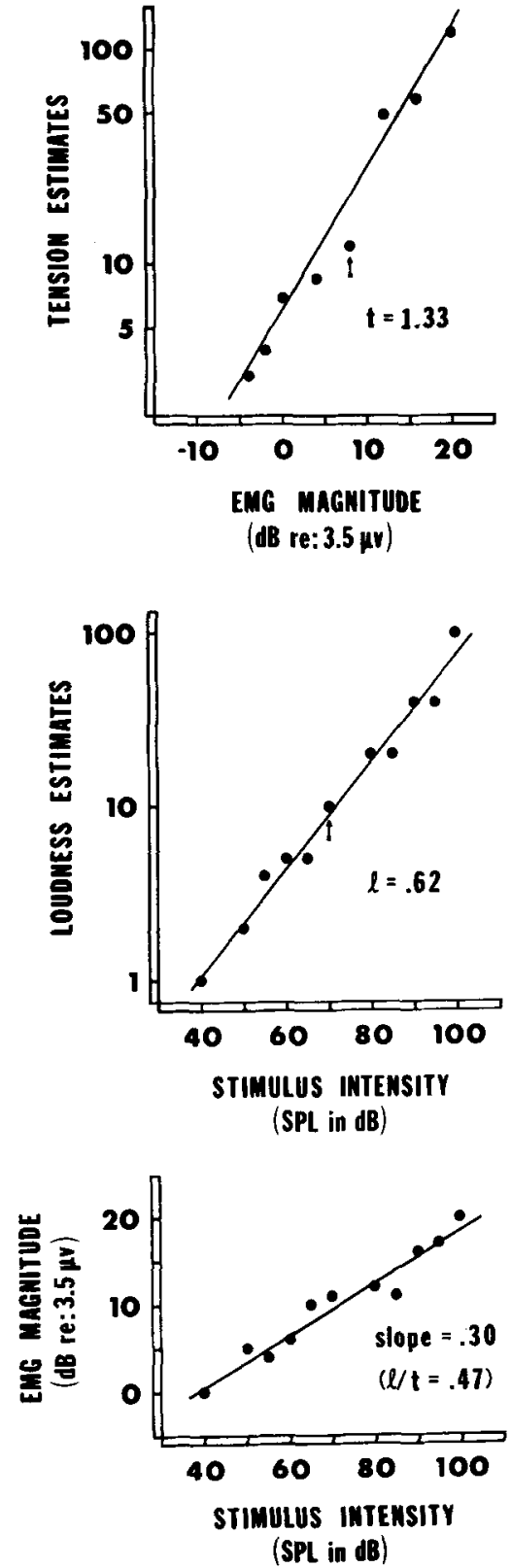

Fig. 3. Same as Fig. 2, except for another $\mathrm{O}$ (JCJB), who used adductive tension of the little finger muscles.

\section{LOUDNESS ESTIMATION}

\section{Subjects}

The Ss were described in the section on tension estimation.

\section{Apparatus}

Auditory stimuli were generated and controlled by standard (Hewlett-Packard and Grason-Stadler) apparatus.

\section{Procedure}

After a 15-min break between the tension and loudness estimation procedures, the $S$ returned to the shielded booth, and was given instructions derived from those used by Stevens (1956). On 
each of 11 trials the $S$ presented himself first with the modulus ( $70 \mathrm{~dB}$ re: $2 \times 10^{-5} \mathrm{~N} / \mathrm{m}^{2}$ ), called " 10 ," and then with a comparison stimulus drawn at random from a set of 11 tone intensities. The stimuli, delivered binaurally, were $1,000-\mathrm{Hz} 1-\mathrm{sec}$ tones with rise and decay times of $25 \mathrm{msec}$. The $\mathrm{S}$ reported on each trial a number which stood in ratio to "10" as the loudness of the comparison stood in ratio to the loudness of the modulus. The intertrial interval was $30 \mathrm{sec}$.

\section{Results}

Table 1 displays the exponents of the power functions fitted by the method of least squares to the loudness estimation data. For all Ss, the exponents were somewhat smaller than would be expected on the basis of previous reports: the average exponent here was 0.55 , whereas Stevens (1966) suggests that the exponent for loudness is approximately 0.64. Despite the discrepancy in exponents, the loudness data, as shown in the middle panels of Figs. 2 and 3 , appear to be adequately fitted by power functions, even for naive Ss giving a single estimate for each tone intensity.

\section{MATCHING TENSION TO LOUDNESS}

\section{Subjects and Apparatus}

The Ss and apparatus were described above.

\section{Procedure}

After a 15-min break, the electrodes were checked for continuity and the $S$ was returned to the shielded booth. Eleven trials were given at $30-\mathrm{sec}$ intervals. On each trial, the $S$ presented himself with one of the tones previously used during loudness estimation. After listening to the tone, the $S$ pressed against the appropriate scaling bar until he felt that the tension produced matched the apparent loudness of the tone. At this point he signaled the $\mathrm{E}$ verbally and waited for instructions to relax. EMG magnitude was recorded at the S's signal unless waveform observations made it necessary to repeat the trial later in the series.

\section{Results}

The least-squares exponents obtained when power functions were individually fitted to the cross-modality matching data are listed in the last column of Table 1. They may be compared to the values in the adjacent column, which are the exponents predicted for the matching functions under the rationale first described by Stevens (1959). In terms of the present study, it can be summarized as follows: If perceived tension is a power function of EMG magnitude with an exponent $t$

$$
\text { Tension }=(\text { EMG Magnitude })^{t}
$$

and if subjective loudness is also related to its physical stimulus by a power function, but with an exponent $\ell$

$$
\text { Loudness }=(\text { Tone Intensity })^{\ell}
$$

then when tension values are equated to values of loudness at a number of levels during the matching procedure, the prediction is that EMG magnitude will be related to tone intensity by a power function whose exponent is the ratio of the two original exponents:

\section{EMG Magnitude $=(\text { Tone Intensity })^{\ell / t}$}

In other words, in log-log coordinates the matching function should be a straight line whose slope is $\ell / t$. The lower panels of Figs. 2 and 3 show that to a first approximation this is, in fact, the case. However, as shown in Table 1, the obtained exponents (slopes) are all smaller than those predicted. Still, for Ss who scaled and then matched abductive tension to loudness, the difference is acceptably small-about 0.04. For those who worked with adductive tension, however, the difference is large-about 0.16-and apparently reliable, since in subsequent replications of the present study with one $S$ (PDS) we obtained first a difference of 0.10 and then one of 0.18 .

\section{DISCUSSION}

Although the results of the tension estimation procedure suggest that perceived muscular tension can now be added to the ever-growing list of perceptual continua that follow a power law (cf. 'Stevens, 1961; Stevens \& Galanter, 1957), a word of caution is in order regarding their interpretation. Salient here is the familiar distinction between operations performed and mechanisms studied; that is, between the psychophysics and the psychophysiology of muscular tension. Operationally taken, our data provide a scale of perceived tension: we instructed our Ss to attend to and to judge muscular tension; we measured the magnitude of muscular contractions. Our operations, however, did not restrict the $S$ to judgments based solely on proprioceptive information. While it seems unlikely that the estimates were mediated by pressure receptors-we have not observed any striking deviations from the power functions at EMG magnitudes thought to be too small to displace the little finger-we cannot reject this possibility out of hand. Similarly, we cannot reject the possibility that efferent rather than afferent information mediated the judgments of tension (cf. Merton, 1964).

Whatever the mechanism, it appears not only that perceived tension grows as a power function of the magnitude of sustained muscular contractions, but also that the exponent may depend on the nature of the muscle under study. To be sure, this seems plausible since muscles vary in a number of ways: in their placement with respect to the receptors that transduce their output (presuming here afferent mediation); and (presuming instead efferent mediation) in their innervation ratios, that is, in the number of muscle fibers served by a motoneuron (Basmajian, 1967). Given variation of this sort, the possibility that the exponent depends on the muscle would seem to follow as a consequence of Stevens's suggestion that the nature of the sensory system determines the value of the exponent (Stevens, 1961). For the moment, however, the possibility has more logical than experimental support.

The failure to validate the scale for adductive tension deserves comment. The problem seems to be this: although a $\mathbf{S}$ asked to produce adductive tension can do so in at least two ways-he can use his forearm muscles and/or the muscle of his hand-we were prepared to measure the EMG for only one of these ways. Since there is no reason to expect validity of a psychophysical scale whose physical variable may have been incompletely measured, the failure to validate the scale for adductive tension is not surprising. However, no such problem existed for abductive tension: only one muscle was likely to be used under the conditions of the present study, and, with its output measured, validation would be expected and, in fact, was obtained.

\section{REFERENCES}

ALLPORT, F. H. Theories of perception and the concept of structure. New York: Wiley, 1955.

BASMAJIAN, J. V. Muscles alive: Their functions revealed by electromyography. Baltimore: Williams \& Wilkins, 1967.

BRUNO, L. J. J., DAVIDOWITZ, J., \& HEFFERLINE, R. F. EMG waveform duration: A validation method for the surface electromyogram. Behavior Research Methods \& Instrumentation, 1970, 2, 211-219.

HEFFERLINE, R. F. The role of proprioception in the control of behavior. Transactions of the New York Academy of Sciences, 1958, 20, 739-764.

HEFFERLINE, R. F. Learning theory and clinical psychology-An eventual symbiosis. In A. J. Bachrach (Ed.), Experimental foundations of clinical psychology. New York: Basic Books, 1962. Pp. 97-138.

LIPPOLD, O. C. J. The relation between integrated action potentials in a human muscle and its isometric tension. Journal of Physiology, 1952, 117, 492-499. 
LIPPOLD, O. C. J. Electromyography. In P. H. Venables and 1. Martin (Eds.), $A$ manual of psychophysiological methods. New York: Wiley, 1967. Pp. 245-297.

MERTON, P. A. Human position sense and sense of effort. In Symposia of the Society for Experimental Biology. No. XVIII. Homeostasis and feedback mechanisms. Cambridge: Cambridge University Press, 1964. Pp. 387-400.

MOUNTCASTLE, V. B. Neural replication of somatic sensory events. In J. C. Eccles (Ed.), Brain and conscious experience. New York: Springer Verlag, 1966. Pp. 85-109.

STEVENS, J. C., \& MACK, J. D. Scales of apparent force. Journal of Experimenal Psychology, 1959, 58, 405-413.

STEVENS, S. S. The direct estimation of sensory magnitudes-loudness. American Journal of Psychology, 1956, 69, 1-25.

STEVENS, S.S. Cross-modality validation of subjective scales for loudness, vibration, and electric shock. Journal of Experimental Psychology, 1959, 57, 201-209.

STEVENS, S. S. The psychophysics, of sensory function. In W. A. Rosenblith (Ed.), Sensory communication. Cambridge, Mass: M.I.T. Press, 1961. Pp. 1-33.

STEVENS, S. S. Matching functions between loudness and ten other continua. Perception \& Psychophysics, 1966, 1, 5-8.

STEVENS, S. S., \& GALANTER, E. H. Ratio scales and category scales for a dozen perceptual continua. Journal of Experimental Psychology, 1957, 54, 377-411.

WHATMORE, G. B., \& KOHLI, D. R. A neurophysiologic factor in functional disorders. Behavioral Science, 1968, 13, 102-124.

WOOD, H. Psychophysics of active kinesthesis. Journal of Experimental Psychology, 1969, $79,480-485$.

\section{NOTE}

1. The bars also provided the circumstances under which strong cutaneous sensations might be generated. To be sure, this was expected for moderate-to-strong contractions. However, for weak contractions, involving the activation of no more than one or two motor units, it seemed unlikely that enough force would be generated to stimulate cutaneous receptors (cf. Basmajian, 1967, p. 12).

(Accepted for publication November 16, 1970.) 